\title{
A NOTABLE FAMILY OF ENTIRE INTRINSIC MINIMAL GRAPHS IN THE HEISENBERG GROUP WHICH ARE NOT PERIMETER MINIMIZING
}

\author{
D. DANIELLI, N. GAROFALO, AND D. M. NHIEU
}

\begin{abstract}
One of the main objectives of this paper is to unravel a new interesting phenomenon of the sub-Riemannian Bernstein problem with respect to its Euclidean ancestor, with the purpose of also indicating a possible line of attack toward its solution. We show that the global intrinsic graphs (1.2) are unstable critical points of the horizontal perimeter. As a consequence of this fact, the study of the stability acquires a central position in the problem itself.
\end{abstract}

\section{Introduction}

The development of geometric measure theory in sub-Riemannian spaces has received a strong impulse over the past decade, see [Pa1, [Pa2, [CDG], [KR], [E1], [E2], E3], Gro, [GN], Be], DS, DGN1, [AK1, AK2, CS1, A], FSS1, Ma1, FSS2, Ma2, CMS, FSS3, BRS, [DGN4, DGN5], [L], LM], [FSS4, [Ma3, CS2 [P1], P2], GP], [CG], CHMY], CH], [HP1], [HP2], RR, [BC], Se1], Se2], [Mo. In particular, the papers [GP, [CHMY] and [CH] contain a detailed study of the Bernstein problem in the first Heisenberg group $\mathbb{H}^{1}$, and in more general CR manifolds of real dimension three. Despite the progress made in these latter papers, this problem presently still constitutes a basic open question. One of the main objectives of this paper is to unravel a new interesting phenomenon of the sub-Riemannian Bernstein problem with respect to its Euclidean ancestor, with the purpose of also indicating a possible line of attack toward its solution.

To provide the reader with some perspective, we recall that in the Heisenberg group $\mathbb{H}^{n}$ a basic discovery of Franchi, Serapioni and Serra Cassano is a structure theorem à la De Giorgi for sets of locally finite horizontal perimeter [FSS1] (see [FSS3 for an extension to Carnot groups of step $r=2$ ). To prove the latter they show that the non-isotropic blow-up of such a set at a point of its reduced boundary produces a vertical hyperplane

$$
P_{\gamma}=\left\{(x, y, t) \in \mathbb{H}^{n} \mid<a, x>+<b, y>=\gamma\right\}, \quad a^{2}+b^{2} \neq 0,
$$

(when $\gamma=0$ these sets are also the maximal subgroups of $\mathbb{H}^{n}$ ). Recalling that the characteristic locus of a hypersurface $\mathcal{S} \subset \mathbb{H}^{n}$, denoted henceforth by $\Sigma(\mathcal{S})$, is the collection of all points $g \in \mathcal{S}$ at which $T_{g} \mathcal{S}=H_{g} \mathbb{H}^{n}$, where $H \mathbb{H}^{n}$ denotes the horizontal bundle of $\mathbb{H}^{n}$, it is easy to recognize that $\Sigma\left(P_{\gamma}\right)=\varnothing$ for any $\gamma \in \mathbb{R}$. Therefore, in analogy with the Euclidean situation, the cited blow-up result from [FSS1] suggests the natural conjecture that if $\mathcal{S} \subset \mathbb{H}^{n}$ is a $C^{2}$ entire $H$-minimal graph over some hyperplane, and if $\Sigma(\mathcal{S})=\varnothing$, then $\mathcal{S}=P_{\gamma}$ for some $\gamma$. Here, following a perhaps unfortunate tradition of the classical situation, $H$-minimal is intended in the sense that $\mathcal{S}$ is of class $C^{2}$, and the horizontal mean curvature, defined in (2.13) below, vanishes identically as a continuous function on $\mathcal{S}$. However, for $\mathbb{H}^{n}$ the situation is very different

Date: October 8, 2018.

Key words and phrases. $H$-minimal surfaces. Intrinsic graphs. First and second variation formulas.

First author supported in part by NSF CAREER Grant, DMS-0239771.

Second author supported in part by NSF Grant DMS-0300477. 
than in Euclidean space. In fact, it was proved in GP that there exist non-planar entire $H$ minimal graphs with empty characteristic locus, thus violating the above plausible conjecture. For instance, the non-planar real analytic surfaces

$$
\mathcal{S}=\left\{(x, y, t) \in \mathbb{H}^{1} \mid x=y(\alpha t+\beta), \alpha>0, \beta \in \mathbb{R}\right\},
$$

are $H$-minimal, and they have empty characteristic locus. Moreover, the surfaces (1.2) are global intrinsic $X_{1}$-graphs in the sense of Franchi, Serapioni and Serra Cassano, see FSS4 (we stress that intrinsic graphs have empty characteristic locus by definition). This means that there exists a globally defined function $\phi: \mathbb{R}_{u, v}^{2} \rightarrow \mathbb{R}$ such that, in the coordinates $(x, y, t)$, we can parameterize (1.2) as follows

$$
(x, y, t)=(0, u, v) \circ \phi(u, v) e_{1}=(0, u, v) \circ(\phi(u, v), 0,0)=\left(\phi(u, v), u, v-\frac{u}{2} \phi(u, v)\right),
$$

where $\circ$ indicates the non-Abelian group multiplication in $\mathbb{H}^{1}$, see (2.1). In fact, imposing the defining equation $x=y(\alpha t+\beta)$ for $\mathcal{S}$, with $\alpha>0$, we obtain the function

$$
\phi(u, v)=\frac{2 u(\alpha v+\beta)}{2+\alpha u^{2}}
$$

which describes $\mathcal{S}$ as an entire $X_{1}$-graph. We also note that a vertical plane $P_{\gamma}$ is a global $X_{1}$-graph if $a \neq 0$ (or an $X_{2}$-graph if $b \neq 0$ ), with

$$
\phi(u, v)=\frac{\gamma-b u}{a}, \quad \text { if } \quad a \neq 0 .
$$

Examples such as (1.2) seem to cast a dim light on the Bernstein problem in $\mathbb{H}^{1}$. There is however a deeper aspect of the problem which has gone unnoticed so far. What could be happening in fact is that, due to the different nature of the relevant perimeter functional, global intrinsic graphs such as (1.2) are only stationary for the horizontal perimeter, but not stable. In this paper we examine this aspect in depth. More precisely, we recall that, thanks to the convexity of the area functional

$$
\mathcal{A}(u)=\int_{\Omega} \sqrt{1+|D u|^{2}} d x, \quad \Omega \subset \mathbb{R}^{n},
$$

in the classical theory of minimal surfaces any critical point of $\mathcal{A}$ is automatically stable, i.e., it is a local minimizer, see e.g. [CM]. By contrast, we show that the global intrinsic graphs (1.2) are unstable critical points of the horizontal perimeter $P_{H}(\mathcal{S})$ defined in (2.22) below. As a consequence of this fact, the study of the stability acquires a central position in the problem itself, and our results suggest that, if properly understood from this new perspective, the Bernstein property is still true in $\mathbb{H}^{1}$. Besides their intrinsic interest, we believe that the relevance of our results lies in the method of proof, which is quite general and flexible, and has the potential of being successfully applied to attack the sub-Riemannian Bernstein problem. In Geometric Measure Theory there exist in essence two (different, but equivalent) approaches to stability: the former is based on the so-called method of calibrations, the latter on second variation formulas. Our approach revolves around a general second variation formula established in [DGN3], and on the explicit construction of a continuum of directions along which the intrinsic perimeter strictly decreases. We need to introduce a basic definition.

Definition 1.1. We say that a $C^{2}$ oriented $H$-minimal surface $\mathcal{S} \subset \mathbb{H}^{1}$, with $\Sigma(\mathcal{S})=\varnothing$, is stationary if it has vanishing first variation of the $H$-perimeter, i.e., if

$$
\left.\mathcal{V}_{I}^{H}(\mathcal{S} ; \mathcal{X}) \stackrel{\text { def }}{=} \frac{d}{d \lambda} P_{H}\left(\mathcal{S}^{\lambda}\right)\right|_{\lambda=0}=0
$$


for any deformation $\mathcal{S} \rightarrow \mathcal{S}^{\lambda}=\mathcal{S}+\lambda \mathcal{X}$, where $\mathcal{X} \in C_{0}^{2}\left(\mathcal{S}, \mathbb{H}^{1}\right)$, with $\mathcal{X} \not \equiv 0$. We say that a stationary $\mathcal{S}$ is stable if the second variation is nonnegative, i.e.,

$$
\left.\mathcal{V}_{I I}^{H}(\mathcal{S} ; \mathcal{X}) \stackrel{\text { def }}{=} \frac{d^{2}}{d \lambda^{2}} P_{H}\left(\mathcal{S}^{\lambda}\right)\right|_{\lambda=0} \geq 0
$$

for any $\mathcal{X} \in C_{0}^{2}\left(\mathcal{S}, \mathbb{H}^{1}\right)$, with $\mathcal{X} \not \equiv 0$. If there exists such an $\mathcal{X}$ for which $\mathcal{V}_{I I}^{H}(\mathcal{S} ; \mathcal{X})<0$, then we say that $\mathcal{S}$ is unstable.

It has been proved in DGN3 that $\mathcal{S}$ is stationary if and only if $\mathcal{S}$ is $H$-minimal, see Theorem 3.1 below. In this paper we establish the following result.

Theorem 1.2. For every $\alpha>0$ and $\beta \in \mathbb{R}$, the $H$-minimal global intrinsic $X_{1}$-graphs

$$
\mathcal{S}=\left\{(x, y, t) \in \mathbb{H}^{1} \mid x=y(\alpha t+\beta)\right\},
$$

are unstable. More precisely, there exist $a \in C_{0}^{\infty}(\mathcal{S}), a \not \equiv 0$, and $h \in C_{0}^{\infty}(\mathcal{S}), h \not \equiv 0$, such that with either $\mathcal{X}=a X_{1}$, or $\mathcal{X}=h \boldsymbol{\nu}_{H}$, we have $\mathcal{V}_{I I}^{H}(\mathcal{S} ; \mathcal{X})<0$.

To explain the strategy behind Theorem 1.2 we mention that in Section 2 we collect some preliminary material which constitutes the geometric backbone of the paper. The novel part of the paper is contained in Section [3. An essential ingredient in the proof of Theorem [1.2 is the second variation formula, see Theorem 3.2 below. Combining the latter with some basic sub-Riemannian integration by parts formulas, see Lemmas 3.5] and [3.6. we reduce the study of the stability of (1.2) to checking the validity of some Hardy type inequalities on $\mathcal{S}$, see Lemmas 3.7 and 3.9. Using the representation of $\mathcal{S}$ as a global graph, we then pull back such Hardy inequalities to ones onto the $(y, t)$-plane, see Lemma 3.12 Finally, in Lemma 3.13 and Corollary 3.14 we explicitly construct the directions such that, deforming the surface (1.2) along them, the $H$-perimeter strictly decreases. This establishes the instability of (1.2).

We would like to close this introduction with some conjectures which are suggested by the present work. Consider a $C^{2}, H$-minimal intrinsic $X_{1}$-graph $\mathcal{S} \subset \mathbb{H}^{1}$. Denoting by $\mathcal{B}_{\phi}$ the linearized Burger's operator, whose action on a function $F=F(u, v)$ is given by $\mathcal{B}_{\phi}(F)=$ $F_{u}+\phi F_{v}$, then it was proved in Theorem $1.2 \mathrm{in}$ [ASV] that, provided that $\phi \in C_{0}^{2}\left(\mathbb{R}^{2}\right)$, the $H$-perimeter of $\mathcal{S}$ is given by

$$
P_{H}(\mathcal{S})=\int_{\text {supp }(\phi)} \sqrt{1+\mathcal{B}_{\phi}(\phi)^{2}} d u \wedge d v
$$

Now, if we think of (1.7) as a functional $P_{H}(\phi)$ acting on $\phi$, one can easily recognize that, given $\zeta \in C_{0}^{\infty}\left(\mathbb{R}^{2}\right)$, then the first variation of $P_{H}(\mathcal{S})$ with respect to the deformation $\mathcal{S} \rightarrow \mathcal{S}^{\lambda}=\mathcal{S}+\lambda \mathcal{X}$, with $\mathcal{X}=\zeta X_{1}$, is given by

$$
\left.\mathcal{V}_{I}^{H}(\mathcal{S} ; \mathcal{X}) \stackrel{\text { def }}{=} \frac{d P_{H}\left(\mathcal{S}^{\lambda}\right)}{d \lambda}\right|_{\lambda=0}=\int_{\mathbb{R}^{2}} \frac{\mathcal{B}_{\phi}(\phi)}{\sqrt{1+\mathcal{B}_{\phi}(\phi)^{2}}}\left(\zeta_{u}+\phi \zeta_{v}+\zeta \phi_{v}\right) d u \wedge d v .
$$

In view of Theorem [1.2 it is natural to make the following conjecture: Suppose that $\phi$, belonging to a suitable Sobolev space, is a local (or even a global) minimizer of (1.7), and therefore in particular also a critical point, then after modification on a set of measure zero, $\phi$ must be of the type (1.5).

If we assume a priori that $\phi \in C^{2}\left(\mathbb{R}^{2}\right)$, then integrating by parts in (1.8) we obtain that

$$
\mathcal{V}_{I}^{H}(\mathcal{S} ; \mathcal{X})=-\int_{\mathbb{R}^{2}} \zeta \mathcal{B}_{\phi}\left(\frac{\mathcal{B}_{\phi}(\phi)}{\sqrt{1+\mathcal{B}_{\phi}(\phi)^{2}}}\right) d u \wedge d v, \quad \zeta \in C_{0}^{\infty}\left(\mathbb{R}^{2}\right)
$$


On the other hand, under the same regularity hypothesis on $\phi$ one can recognize, see [GS], that

$$
\mathcal{B}_{\phi}\left(\frac{\mathcal{B}_{\phi}(\phi)}{\sqrt{1+\mathcal{B}_{\phi}(\phi)^{2}}}\right)=-\mathcal{H},
$$

where $\mathcal{H}$ represents the $H$-mean curvature of $\mathcal{S}$, defined in (2.13) below. Therefore, a $C^{2}$ global intrinsic graph $\mathcal{S}$ is a critical point of (1.7) if and only if $\mathcal{S}$ is $H$-minimal. As a consequence of these considerations, in the $C^{2}$ framework the above conjecture could be reformulated by saying that: The only $C^{2}$, stable, global intrinsic graphs in $\mathbb{H}^{1}$ are the vertical planes. Finally, we would also like to return to the conjecture in the opening of this introduction and amend it as follows: In $\mathbb{H}^{1}$ the vertical planes (1.1) are the only $C^{2}$, stable, entire $H$-minimal graphs (over some plane).

Acknowledgment: The problems treated in this paper were inspired by some stimulating discussions with F. Serra Cassano and R. Serapioni during a visit of the second named author at the University of Trento in April 2005. He would like to thank them for their gracious hospitality. The authors would also like to thank the anonymous referee for his/her careful reading of the manuscript and for some comments which helped to improve the presentation of the paper.

\section{Preliminary material}

In this section we introduce some relevant notation and definitions from DGN3 which will be used in the proof of Theorem 1.2. We consider the first Heisenberg group $\mathbb{H}^{1}=\left(\mathbb{R}^{3}, \circ\right)$ with group law

$$
(x, y, t) \circ\left(x^{\prime}, y^{\prime}, t^{\prime}\right)=\left(x+x^{\prime}, y+y^{\prime}, t+t^{\prime}+\frac{1}{2}\left(x y^{\prime}-x^{\prime} y\right)\right),
$$

and non-isotropic dilations $\delta_{\lambda}(x, y, t)=\left(\lambda x, \lambda y, \lambda^{2} t\right)$, see $[\underline{S}$. Hereafter, we will use the letters $g=(x, y, t), g^{\prime}=\left(x^{\prime}, y^{\prime}, t^{\prime}\right)$, etc., to indicate points in $\mathbb{H}^{1}$. Denoting with $\left(L_{g}\right)_{*}$ the differential of the left-translation operator $L_{g}: \mathbb{H}^{1} \rightarrow \mathbb{H}^{1}$ defined by $L_{g}\left(g^{\prime}\right)=g \circ g^{\prime}$, and letting $e_{i}, i=1,2,3$, indicate the standard orthonormal basis of $\mathbb{R}^{3}$, one readily verifies that

$$
\left\{\begin{array}{l}
X_{1}(g) \stackrel{\text { def }}{=}\left(L_{g}\right)_{*}\left(e_{1}\right)=\frac{\partial}{\partial x}-\frac{y}{2} \frac{\partial}{\partial t}, \\
X_{2}(g) \stackrel{\text { def }}{=}\left(L_{g}\right)_{*}\left(e_{2}\right)=\frac{\partial}{\partial y}+\frac{x}{2} \frac{\partial}{\partial t}, \\
T(g) \stackrel{\text { def }}{=}\left(L_{g}\right)_{*}\left(e_{3}\right)=\frac{\partial}{\partial t} .
\end{array}\right.
$$

The three vector fields $\left\{X_{1}, X_{2}, T\right\}$ generate the Lie algebra $\mathfrak{h}^{1}$ of all left-invariant vector fields on $\mathbb{H}^{1}$. They satisfy at every point of $\mathbb{H}^{1}$ the non-trivial commutation relation

$$
\left[X_{1}, X_{2}\right]=T,
$$

all other commutators being trivial. In view of (2.3), the Heisenberg group constitutes the first (and perhaps most important) prototype of a class of graded nilpotent Lie groups nowadays known as Carnot groups, see [Fo], [S], Gro, [Pa2], Be]. We observe explicitly that, if we let $V_{1}=\mathbb{R}_{x, y}^{2} \times\{0\}_{t}$, and $V_{2}=\{0\}_{x, y} \times \mathbb{R}_{t}$, then the Heisenberg algebra admits the decomposition $\mathfrak{h}^{1}=V_{1} \oplus V_{2}$. We assume hereafter that $\mathbb{H}^{1}$ be endowed with a left-invariant Riemannian metric with respect to which $\left\{X_{1}, X_{2}, T\right\}$ constitute an orthonormal basis. The inner product with respect to this metric will be denoted by $\langle\cdot, \cdot\rangle$. This is the only inner product that will be used in this paper, therefore there will be no confusion with other inner products, such as for 
instance the Euclidean one, in $\mathbb{R}^{3}$. The corresponding Levi-Civita connection on $\mathbb{H}^{1}$ will be denoted by $\nabla_{X} Y$. We will denote by $H \mathbb{H}^{1}$ the subbundle of the tangent bundle $T \mathbb{H}^{1}$ generated by the distribution $\left\{X_{1}, X_{2}\right\}$. The horizontal Levi-Civita connection is given as follows. For any $X \in \Gamma\left(T \mathbb{H}^{1}\right), Y \in \Gamma\left(H \mathbb{H}^{1}\right)$ we let

$$
\nabla_{X}^{H} Y=\sum_{i=1}^{2}<\nabla_{X} Y, X_{i}>X_{i},
$$

and one can easily verify that $\nabla_{X}^{H} Y$ is metric preserving and torsion free, in the sense that if we define the horizontal torsion of $\mathcal{S}$ as

$$
T^{H}(X, Y)=\nabla_{X}^{H} Y-\nabla_{Y}^{H} X-[X, Y]^{H},
$$

where $[X, Y]^{H}=\sum_{i=1}^{2}<[X, Y], X_{i}>X_{i}$, then $T^{H}(X, Y)=0$. Given a function $f \in C^{1}\left(\mathbb{H}^{1}\right)$, its Riemannian gradient is given by

$$
\nabla f=X_{1} f X_{1}+X_{2} f X_{2}+T f T,
$$

whereas its horizontal gradient is given by the projection of $\nabla f$ onto the subbundle $\mathbb{H}^{1}$, hence

$$
\nabla^{H} f=<\nabla f, X_{1}>X_{1}+<\nabla f, X_{2}>X_{2}=X_{1} f X_{1}+X_{2} f X_{2} .
$$

Given an oriented $C^{2}$ surface $S \subset \mathbb{H}^{1}$, we denote by $N$ its (non-unit) Riemannian normal with respect to the orthonormal frame $\left\{X_{1}, X_{2}, T\right\}$, and by $\boldsymbol{\nu}=\boldsymbol{N} /|\boldsymbol{N}|$ its Riemannian Gauss map. We consider the quantities

$$
p=\left\langle\boldsymbol{N}, X_{1}>, \quad q=\left\langle\boldsymbol{N}, X_{2}>, \quad \omega=<\boldsymbol{N}, T>, \quad W=\sqrt{p^{2}+q^{2}} .\right.\right.
$$

In this paper we adopt the classical non-parametric point of view, see for instance [MM], according to which a $C^{2}$ surface $\mathcal{S} \subset \mathbb{H}^{1}$ is a subset of $\mathbb{R}^{3}$ which locally coincides with the zero set of a real function. Thus, for every $g_{0} \in \mathcal{S}$ there exists an open set $\mathcal{O} \subset \mathbb{H}^{1}$ and a function $\phi \in C^{2}(\mathcal{O})$ such that: (i) $|\nabla \phi(g)| \neq 0$ for every $g \in \mathcal{O}$; (ii) $\mathcal{S} \cap \mathcal{O}=\{g \in \mathcal{O} \mid \phi(g)=0\}$. We will always assume that $\mathcal{S}$ is oriented in such a way that for every $g \in \mathcal{S}$ one has

$$
\mathbf{N}(g)=\nabla \phi(g)=X_{1} \phi(g) X_{1}+X_{2} \phi(g) X_{2}+T \phi(g) T .
$$

We note explicitly that, in this situation, the functions $p, q, \omega$ defined in (2.5), which are given by $p=X_{1} \phi, q=X_{2} \phi, \omega=T \phi$, are not only defined on $\mathcal{S}$, but for every $g_{0} \in \mathcal{S}$ they belong to $C^{1}(\mathcal{O})$. This notion of $C^{2}$ surface obviously includes the entire intrinsic graphs considered in Theorem 1.2. In fact, in the case of the surfaces $\mathcal{S}$ in (1.6), we have (see (3.17)),

$$
p=X_{1} \phi=1+\frac{\alpha}{2} y^{2}, \quad q=X_{2} \phi=-\alpha t-\beta-\frac{\alpha}{2} x y, \quad \omega=T \phi=-\alpha y,
$$

and thus in particular the field $\boldsymbol{N}=p X_{1}+q X_{2}+\omega T$ belongs to $C^{\infty}\left(\mathcal{O}, \mathbb{H}^{1}\right)$, with $\mathcal{O}=\mathbb{H}^{1}$.

We emphasize here that the local defining function $\phi$ in (i) and (ii) above has a different meaning from the function $\phi$ in the definition of intrinsic graph in the introduction. Given a surface $\mathcal{S} \subset \mathbb{H}^{1}$, on the set $\mathcal{S} \backslash \Sigma(\mathcal{S})$ we define the horizontal Gauss map by

$$
\boldsymbol{\nu}_{H}=\bar{p} X_{1}+\bar{q} X_{2},
$$

where we have let

$$
\text { 7) } \bar{p}=\frac{p}{W}, \quad \bar{q}=\frac{q}{W}, \quad \text { so that } \quad\left|\boldsymbol{\nu}_{H}\right|^{2}=\bar{p}^{2}+\bar{q}^{2} \equiv 1 \quad \text { on } \quad \mathcal{S} \backslash \Sigma(\mathcal{S}) \text {. }
$$

Given a point $g_{0} \in \mathcal{S} \backslash \Sigma(\mathcal{S})$, the horizontal tangent space of $\mathcal{S}$ at $g_{0}$ is defined by

$$
H T_{g_{0}}(\mathcal{S})=\left\{\boldsymbol{v} \in H_{g_{0}} \mathbb{H}^{1} \mid<\boldsymbol{v}, \boldsymbol{\nu}_{H}\left(g_{0}\right)>=0\right\} .
$$

Let us notice that a basis for $H T_{g_{0}}(\mathcal{S})$ is given by the vector field

$$
\boldsymbol{\nu}_{H}^{\perp}=\bar{q} X_{1}-\bar{p} X_{2} \text {. }
$$


Given a function $u \in C^{1}(\mathcal{S})$ one clearly has $\nabla^{H, \mathcal{S}} u\left(g_{0}\right) \in H T_{g_{0}}(\mathcal{S})$. We next recall some basic definitions from DGN3.

Definition 2.1. Let $\mathcal{S} \subset \mathbb{H}^{1}$ be a $C^{2}$ surface, with $\Sigma(\mathcal{S})=\varnothing$, then we define the horizontal connection on $\mathcal{S}$ as follows. For every $X, Y \in C^{1}(\mathcal{S} ; H T \mathcal{S})$ we let

$$
\nabla_{X}^{H, \mathcal{S}} Y=\nabla \frac{H}{X} \bar{Y}-<\nabla \frac{H}{X} \bar{Y}, \nu_{H}>\nu_{H},
$$

where $\bar{X}, \bar{Y} \in C^{1}\left(\mathbb{H}^{1} ; H \mathbb{H}^{1}\right)$ are such that $\bar{X}=X, \bar{Y}=Y$ on $\mathcal{S}$.

Similarly to the Riemannian case, it is possible to prove that $\nabla_{X}^{H, \mathcal{S}} Y$ does not depend on the extensions $\bar{X}, \bar{Y}$. The tangential horizontal gradient of a function $f \in C^{1}(\mathcal{S})$ is defined as follows

$$
\nabla^{H, \mathcal{S}} f=\nabla^{H} \bar{f}-<\nabla^{H} \bar{f}, \boldsymbol{\nu}_{H}>\boldsymbol{\nu}_{H},
$$

where $\bar{f}$ denotes any extension of $f$ to all of $\mathbb{H}^{1}$. The definition of $\nabla^{H, \mathcal{S}} f$ is well-posed since $\nabla^{H, \mathcal{S}} f$ only depends on the values of $f$ on $\mathcal{S}$. Since $\left|\boldsymbol{\nu}_{H}\right| \equiv 1$ on $\mathcal{S} \backslash \Sigma(\mathcal{S})$, we clearly have $<\nabla^{H, \mathcal{S}} f, \boldsymbol{\nu}_{H}>=0$, and therefore

$$
\left|\nabla^{H, \mathcal{S}} f\right|^{2}=\left|\nabla^{H} \bar{f}\right|^{2}-<\nabla^{H} \bar{f}, \nu_{H}>^{2} .
$$

All the above definitions are specializations to $\mathbb{H}^{1}$ of analogous ones for general Carnot groups, see DGN3]. The next definition contains the essential geometric concept of horizontal second fundamental form. It is convenient to state it for $\mathbb{H}^{n}$, rather than $\mathbb{H}^{1}$.

Definition 2.2. Let $\mathcal{S} \subset \mathbb{H}^{n}$ be a $C^{2}$ hypersurface with $\Sigma(\mathcal{S})=\varnothing$, then for every $X, Y \in$ $C^{1}(\mathcal{S} ; H T \mathcal{S})$ we define a tensor field of type $(0,2)$ on $\mathcal{S}$, as follows

$$
I I^{H, \mathcal{S}}(X, Y)=\left\langle\nabla \frac{H}{X} \bar{Y}, \boldsymbol{\nu}_{H}>\boldsymbol{\nu}_{H},\right.
$$

where $\bar{X}, \bar{Y}$ have the same meaning as in Definition 2.1. We call $I I^{H, \mathcal{S}}(\cdot, \cdot)$ the horizontal second fundamental form of $\mathcal{S}$. We also define $\mathcal{A}^{H, \mathcal{S}}: H T \mathcal{S} \rightarrow H T \mathcal{S}$ by letting for every $g \in \mathcal{S}$ and $\boldsymbol{u}, \boldsymbol{v} \in H T_{g} \mathcal{S}$

$$
<\mathcal{A}^{H, \mathcal{S}} \boldsymbol{u}, \boldsymbol{v}>=-<I I^{H, \mathcal{S}}(\boldsymbol{u}, \boldsymbol{v}), \boldsymbol{\nu}_{H}>=-<\nabla \frac{H}{X} \bar{Y}, \boldsymbol{\nu}_{H}>,
$$

where $X, Y \in C^{1}(\mathcal{S}, H T \mathcal{S})$ are such that $X_{g}=\boldsymbol{u}, Y_{g}=\boldsymbol{v}$, and $\bar{X}, \bar{Y}$ are as above. We call the linear map $\mathcal{A}^{H, \mathcal{S}}: H T_{g} \mathcal{S} \rightarrow H T_{g} \mathcal{S}$ the horizontal shape operator. If $\boldsymbol{e}_{1}, \ldots, \boldsymbol{e}_{2 n-1}$ denotes a local orthonormal frame for $H T \mathcal{S}$, then the matrix of the horizontal shape operator with respect to the basis $\boldsymbol{e}_{1}, \ldots, \boldsymbol{e}_{2 n-1}$ is given by the $(2 n-1) \times(2 n-1)$ matrix $-\left[\left\langle\nabla_{\boldsymbol{e}_{i}}^{H} \boldsymbol{e}_{j}, \boldsymbol{\nu}_{H}\right\rangle\right]_{i, j=1, \ldots, 2 n-1}$.

If $\mathcal{S}$ has non-empty characteristic locus $\Sigma(\mathcal{S})$, then we consider $\mathcal{S}^{\prime}=\mathcal{S} \backslash \Sigma(\mathcal{S})$ and define the $H$-mean curvature of $\mathcal{S}$ at a point $g_{0} \in \mathcal{S}^{\prime}$ as follows

$$
\mathcal{H}=-\operatorname{trace} \mathcal{A}^{H, \mathcal{S}}=-\sum_{j=1}^{2 n-1}<\nabla_{\boldsymbol{e}_{i}}^{H} \boldsymbol{e}_{j}, \boldsymbol{\nu}_{H}>.
$$

We recall that is was proved in $\left[\mathrm{B}\right.$, Ma3 that $\mathcal{H}^{Q-1}(\Sigma(\mathcal{S}))=0$, where $\mathcal{H}^{s}$ denotes the $s$ dimensional Hausdorff measure associated with the horizontal or Carnot-Carathéodory distance of $\boldsymbol{G}$, and $Q$ indicates the homogeneous dimension of $\boldsymbol{G}$. If $g_{0} \in \Sigma(\mathcal{S})$ we let

$$
\mathcal{H}\left(g_{0}\right)=\lim _{g \rightarrow g_{0}, g \in \mathcal{S} \backslash \Sigma(\mathcal{S})} \mathcal{H}(g),
$$

provided that such limit exists, finite or infinite. We do not define the $H$-mean curvature at those points $g_{0} \in \Sigma(\mathcal{S})$ at which the limit does not exist. The following result is taken from DGN3. 
Proposition 2.3. The $H$-mean curvature of $\mathcal{S} \subset \mathbb{H}^{1}$ coincides with the function

$$
\mathcal{H}=\sum_{i=1}^{2} \nabla_{i}^{H, \mathcal{S}}<\boldsymbol{\nu}_{H}, X_{i}>=\nabla_{1}^{H, \mathcal{S}} \bar{p}+\nabla_{2}^{H, \mathcal{S}_{\bar{q}}}=X_{1} \bar{p}+X_{2} \bar{q},
$$

where $\bar{p}, \bar{q}$ are as in (2.7).

Definition 2.4. A $C^{2}$ surface $\mathcal{S} \subset \mathbb{H}^{1}$ is called $H$-minimal if $\mathcal{H} \equiv 0$ as a continuous function on $\mathcal{S}$.

In keeping up with the notation of [DGN3] it will be convenient to indicate with $Y \zeta$ and $Z \zeta$ the respective actions of the vector fields $\boldsymbol{\nu}_{H}$ and $\boldsymbol{\nu}_{H}^{\perp}$ on a function $\zeta \in C_{0}^{1}(\mathcal{S} \backslash \Sigma(\mathcal{S}))$, thus

$$
Y \zeta \stackrel{\text { def }}{=} \bar{p} X_{1} \zeta+\bar{q} X_{2} \zeta, \quad Z \zeta \stackrel{\text { def }}{=} \bar{q} X_{1} \zeta-\bar{p} X_{2} \zeta .
$$

The frame $\{Z, Y, T\}$ is orthonormal. It is worth observing that, since the metric tensor $\left\{g_{i j}\right\}$ with respect to the inner product $\left\langle\cdot, \cdot>\right.$ has the property $\operatorname{det}\left\{g_{i j}\right\}=1$, then the (Riemannian) divergence in $\mathbb{H}^{1}$ of these vector fields is given by

$$
\operatorname{div} Y=X_{1} \bar{p}+X_{2} \bar{q}=\mathcal{H}, \quad \operatorname{div} Z=X_{1} \bar{q}-X_{2} \bar{p} .
$$

Using Cramer's rule one easily obtains from (2.15)

$$
X_{1} \zeta=\bar{q} Z \zeta+\bar{p} Y \zeta, \quad X_{2} \zeta=\bar{q} Y \zeta-\bar{p} Z \zeta .
$$

One also has

$$
\nabla_{1}^{H, \mathcal{S}} \zeta=\bar{q} Z \zeta, \quad \nabla_{2}^{H, \mathcal{S}} \zeta=-\bar{p} Z \zeta
$$

so that

$$
\left|\nabla^{H, \mathcal{S}} \zeta\right|^{2}=(Z \zeta)^{2}
$$

We notice that

$$
\bar{q} Z \bar{p}-\bar{p} Z \bar{q}=\mathcal{H}
$$

This can be easily recognized using Proposition 2.3 and (2.17), as follows

$$
\mathcal{H}=X_{1} \bar{p}+X_{2} \bar{q}=\bar{q} Z \bar{p}-\bar{p} Z \bar{q}+\bar{p} Y \bar{p}+\bar{q} Y \bar{q}=\bar{q} Z \bar{p}-\bar{p} Z \bar{q},
$$

where we have used the fact that $0=\frac{1}{2} Y\left(\bar{p}^{2}+\bar{q}^{2}\right)=\bar{p} Y \bar{p}+\bar{q} Y \bar{q}$. Finally, we will need the following identity

$$
\mathcal{H}^{2}=(Z \bar{p})^{2}+(Z \bar{q})^{2} .
$$

This can be easily proved observing that (2.20) and the identity $\bar{p}^{2}+\bar{q}^{2}=1$ give

$$
\mathcal{H}^{2}=(Z \bar{p})^{2}+\left(Z \bar{q}^{2}\right)-(\bar{p} Z \bar{p}+\bar{q} Z \bar{q})=(Z \bar{p})^{2}+\left(Z \bar{q}^{2}\right) .
$$

In the classical theory of minimal surfaces, the concept of area or perimeter occupies a central position, see DG1, DG2, DCP, G], MM]. In sub-Riemannian geometry there exists an appropriate notion of perimeter. Given an open set $\Omega \subset \mathbb{H}^{1}$ we denote $\mathcal{F}(\Omega)=\left\{\zeta \in C_{0}^{1}\left(\Omega ; H \mathbb{H}^{1}\right) \mid\right.$ $\left.\|\zeta\|_{L^{\infty}(\Omega)} \leq 1\right\}$. A function $u \in L^{1}(\Omega)$ is said to belong to $B V_{H}(\Omega)$ (the space of functions with finite horizontal bounded variation), if

$$
\operatorname{Var}_{H}(u ; \Omega)=\sup _{\zeta \in \mathcal{F}(\Omega)} \int_{\Omega} u d i v_{H} \zeta d g<\infty .
$$


This space becomes a Banach space with the norm $\|u\|_{B V_{H}(\Omega)}=\|u\|_{L^{1}(\Omega)}+\operatorname{Var}_{H}(u ; \Omega)$. Given a measurable set $E \subset \mathbb{H}^{1}$, the $H$-perimeter of $E$ with respect to the open set $\Omega \subset \mathbb{H}^{1}$ is defined as follows, see for instance [CDG], and [GN],

$$
P_{H}(E ; \Omega)=\operatorname{Var}_{H}\left(\chi_{E} ; \Omega\right) .
$$

Given an oriented $C^{2}$ surface $\mathcal{S}$, we will denote by $d \sigma_{H}$ the $H$-perimeter measure concentrated on $\mathcal{S}$. For any Borel subset $E \subset \mathcal{S}$ such that $P_{H}(E)<\infty$, one has

$$
\begin{aligned}
P_{H}(E) & =\int_{E} d \sigma_{H}=\int_{E} \sqrt{<\boldsymbol{\nu}, X_{1}>^{2}+<\boldsymbol{\nu}, X_{2}>^{2}} d \sigma \\
& =\int_{E} \frac{\sqrt{<\boldsymbol{N}, X_{1}>^{2}+<\boldsymbol{N}, X_{2}>^{2}}}{|\boldsymbol{N}|} d \sigma=\int_{E} \frac{W}{|\boldsymbol{N}|} d \sigma,
\end{aligned}
$$

where in the last equality we have used (2.5). We thus obtain from (2.22)

$$
d \sigma_{H}=\frac{W}{|\boldsymbol{N}|} d \sigma
$$

where $d \sigma$ denotes the standard surface measure.

\section{Proof of Theorem 1.2}

This section is devoted to proving Theorem 1.2 In the course of the proof we need to build some auxiliary results which we fell have an independent interest. We begin by recalling the notions of first and second variation of the $H$-perimeter introduced in Definition [1.1. Classical minimal surfaces are critical points of the perimeter (area functional). It is natural to ask what is the connection between the notion of $H$-minimal surface and that of $H$-perimeter. The answer to this question is contained in the following result from DGN3, see also DGN2].

Theorem 3.1. Let $\mathcal{S} \subset \mathbb{H}^{1}$ be an oriented $C^{2}$ surface with $\Sigma(\mathcal{S})=\varnothing$, then

$$
\mathcal{V}_{I}^{H}(\mathcal{S} ; \mathcal{X})=\int_{\mathcal{S}} \mathcal{H} \frac{\cos (\mathcal{X} \angle \boldsymbol{N})}{\cos \left(\boldsymbol{\nu}_{H} \angle \boldsymbol{N}\right)}|\mathcal{X}| d \sigma_{H}
$$

where $\angle$ denotes the angle between vectors in the inner product $\langle\cdot, \cdot\rangle$. In particular, $\mathcal{S}$ is stationary if and only if it is $H$-minimal.

We emphasize that, thanks to the assumption $\Sigma(\mathcal{S})=\varnothing$, the denominator in the integrand in the right-hand side of (3.1) does not vanish on $\mathcal{S}$. We mention that versions of Theorem 3.1 have also been obtained independently by other people. An approach based on motion by $H$-mean curvature can be found in $[\mathrm{BC}]$. When $\mathcal{X}=h \boldsymbol{\nu}_{H}$, then a proof based on CR-geometry can be found in [CHMY.

A central (and more complex) result for this paper is the following theorem established in DGN3]. Recalling the function $\omega$ defined in (2.5), henceforth we let $\bar{\omega}=\omega / W$.

Theorem 3.2. Let $\mathcal{S} \subset \mathbb{H}^{1}$ be a $C^{2}$ oriented surface with $\Sigma(\mathcal{S})=\varnothing$. The second variation of the $H$-perimeter with respect to the deformation $\mathcal{S} \rightarrow \mathcal{S}^{\lambda}=\mathcal{S}+\lambda \mathcal{X}$, with $\mathcal{X}=a X_{1}+b X_{2}+k T \in$ 
$C_{0}^{2}\left(\mathcal{S} ; \mathbb{H}^{1}\right)$, is given by the formula

$$
\begin{aligned}
\mathcal{V}_{I I}^{H}(\mathcal{S} ; \mathcal{X}) & =\int_{\mathcal{S}}\{-2(\bar{p} Z b-\bar{q} Z a)(T k-\bar{\omega} Y k) \\
& +(T a-\bar{\omega} Y a)[-2 \bar{q} Z k-\bar{q}(a \bar{p}+b \bar{q})-\bar{p}(a \bar{q}-b \bar{p})] \\
& +(T b-\bar{\omega} Y b)[2 \bar{p} Z k+\bar{p}(a \bar{p}+b \bar{q})-\bar{q}(a \bar{q}-b \bar{p})] \\
& +2(a \bar{q}-b \bar{p})(\bar{q} Z a-\bar{p} Z b) \bar{\omega} \\
& +(Z a+\bar{\omega} \bar{p} Z k)^{2}+(Z b+\bar{\omega} \bar{q} Z k)^{2} \\
& +\left(a^{2}+b^{2}\right) \bar{\omega}^{2} \\
& +2 \bar{\omega}(a Z a+b Z b)+2 \bar{\omega}^{2}(a \bar{p}+b \bar{q}) Z k \\
& \left.-(\bar{q} Z a-\bar{p} Z b+(a \bar{q}-b \bar{p}) \bar{\omega})^{2}\right\} d \sigma_{H} .
\end{aligned}
$$

Corollary 3.3. If we choose $a \in C_{0}^{\infty}(\mathcal{S}), b \equiv k \equiv 0$, and therefore $\mathcal{X}=a X_{1}$, then the corresponding second variation of the $H$-perimeter is given by

$$
\begin{aligned}
\mathcal{V}_{I I}^{H}(\mathcal{S} ; \mathcal{X}) & =\int_{\mathcal{S}}\left\{\bar{p}^{2}(Z a)^{2}+\bar{p}^{2} \bar{\omega}^{2} a^{2}\right. \\
& \left.+\bar{\omega} Z\left(a^{2}\right)-\bar{p} \bar{q}\left(T\left(a^{2}\right)-\bar{\omega} Y\left(a^{2}\right)\right)\right\} d \sigma_{H} .
\end{aligned}
$$

Proof. It follows in an elementary fashion from (3.2). One only needs to keep in mind that $\bar{p}^{2}+\bar{q}^{2}=1$.

Corollary 3.4. Given a $C^{3}$ oriented surface $\mathcal{S} \subset \mathbb{H}^{1}$, with $\Sigma(\mathcal{S})=\varnothing$, consider the deformation

$$
\mathcal{S}^{\lambda}=\mathcal{S}+\lambda\left(h \boldsymbol{\nu}_{H}+k T\right), \quad h, k \in C_{0}^{2}(\mathcal{S}),
$$

corresponding to the choice $\mathcal{X}=\bar{p} h X_{1}+\bar{q} h X_{2}$ (notice that $a=\bar{p} h, b=\bar{q} h \in C_{0}^{2}(\mathcal{S})$ ). One has

$$
\begin{aligned}
\mathcal{V}_{I I}^{H}(\mathcal{S} ; \mathcal{X}) & =\int_{\mathcal{S}}(Z h+\bar{\omega} Z k)^{2} d \sigma_{H} \\
& +2 \int_{\mathcal{S}} h \mathcal{H}(T k-\bar{\omega} Y k) d \sigma_{H} \\
& +\int_{\mathcal{S}}\left\{\bar{\omega} Z\left(h^{2}\right)+2 \mathcal{A} h Z k+\mathcal{A} h^{2}\right\} d \sigma_{H},
\end{aligned}
$$

where we have set

$$
\mathcal{A}=(\bar{p} T \bar{q}-\bar{q} T \bar{p})+\bar{\omega}(\bar{q} Y \bar{p}-\bar{p} Y \bar{q})+\bar{\omega}^{2} .
$$

Proof. We notice that we presently have

$$
\begin{gathered}
\bar{p} a+\bar{q} b=h, \quad \bar{q} a-\bar{p} b=0, \\
a Z a+b Z b=h Z h, \\
\bar{p} Z b-\bar{q} Z a=h(\bar{p} Z \bar{q}-\bar{q} Z \bar{p})=-h \mathcal{H},
\end{gathered}
$$

where in the last equality we have used the identity (2.20). We also have 


$$
\begin{aligned}
T a-\bar{\omega} Y a & =\bar{p}(T h-\bar{\omega} Y h)+h(T \bar{p}-\bar{\omega} Y \bar{p}), \\
T b-\bar{\omega} Y b & =\bar{q}(T h-\bar{\omega} Y h)+h(T \bar{q}-\bar{\omega} Y \bar{q}), \\
(Z a+\bar{\omega} \bar{p} Z k)^{2} & =\bar{p}^{2}(Z h+\bar{\omega} Z k)^{2} \\
& +(Z \bar{p})^{2} h^{2}+2 \bar{p} Z \bar{p} h(Z h+\bar{\omega} Z k) \\
(Z b+\bar{\omega} \bar{q} Z k)^{2} & =\bar{q}^{2}(Z h+\bar{\omega} Z k)^{2} \\
& +(Z \bar{q})^{2} h^{2}+2 \bar{q} Z \bar{q} h(Z h+\bar{\omega} Z k) .
\end{aligned}
$$

We next observe that

Substituting these formulas in the right-hand side of (3.2), and using (2.21), we reach the desired conclusion.

A different approach to a version of (3.5) based on CR-geometry was found in CHMY. To reduce further the expressions in the right-hand side of (3.3), (3.5) we would like to transform the terms containing the derivatives $Z\left(a^{2}\right), T\left(a^{2}\right), Y\left(a^{2}\right), Z\left(h^{2}\right)$. For this, we will use the following basic integration by parts formulas proved in [DGN3].

Lemma 3.5. Let $\mathcal{S} \subset \mathbb{H}^{1}$ be a $C^{2}$ oriented surface with $\Sigma(\mathcal{S})=\varnothing$. For any $\zeta \in C_{0}^{1}(\mathcal{S})$ one has

$$
\int_{\mathcal{S}} Z \zeta d \sigma_{H}=-\int_{\mathcal{S}} \zeta \bar{\omega} d \sigma_{H}
$$

Lemma 3.6. With $\mathcal{S}$ as in Lemma 3.5, for any function $\zeta \in C_{0}^{1}(\mathcal{S})$ one has

$$
\int_{\mathcal{S}} T \zeta d \sigma_{H}=\int_{\mathcal{S}} Y \zeta \bar{\omega} d \sigma_{H}+\int_{\mathcal{S}} \zeta \bar{\omega} \mathcal{H} d \sigma_{H}
$$

Using Lemma 3.5 we find

$$
\begin{aligned}
& \int_{\mathcal{S}} \bar{\omega} Z\left(a^{2}\right) d \sigma_{H}=\int_{\mathcal{S}} Z\left(\bar{\omega} a^{2}\right) d \sigma_{H}-\int_{\mathcal{S}} a^{2} Z \bar{\omega} d \sigma_{H} \\
& =-\int_{\mathcal{S}} a^{2} \bar{\omega}^{2} d \sigma_{H}-\int_{\mathcal{S}} a^{2} Z \bar{\omega} d \sigma_{H} .
\end{aligned}
$$

From Lemma 3.6 we obtain instead

$$
\begin{aligned}
& -\int_{\mathcal{S}} \bar{p} \bar{q}\left(T\left(a^{2}\right)-\bar{\omega} Y\left(a^{2}\right)\right) d \sigma_{H} \\
& =\int_{\mathcal{S}} a^{2}\{(\bar{p} T \bar{q}+\bar{q} T \bar{p})-\bar{\omega}(\bar{p} Y \bar{q}+\bar{q} Y \bar{p})-\bar{p} \bar{q} \bar{\omega} \mathcal{H}\} d \sigma_{H} .
\end{aligned}
$$

Substituting (3.7), (3.8) into (3.3), and keeping (2.9) in mind, we finally obtain.

Lemma 3.7. Let $\mathcal{S} \subset \mathbb{H}^{1}$ be a $C^{2}$, oriented surface, with $\Sigma(\mathcal{S})=\varnothing$, then the second variation of the $H$-perimeter, with respect to deformation $\mathcal{S}^{\lambda}=\mathcal{S}+\lambda a X_{1}$, is given by

$$
\begin{aligned}
\mathcal{V}_{I I}^{H}\left(\mathcal{S} ; a X_{1}\right) & =\int_{\mathcal{S}} \bar{p}^{2}\left|\nabla^{H, \mathcal{S}} a\right|^{2} d \sigma_{H}+\int_{\mathcal{S}} a^{2}\{(\bar{p} T \bar{q}+\bar{q} T \bar{p})-\bar{\omega}(\bar{p} Y \bar{q}+\bar{q} Y \bar{p}) \\
& \left.-\bar{q}^{2} \bar{\omega}^{2}-Z \bar{\omega}-\bar{p} \bar{q} \bar{\omega} \mathcal{H}\right\} d \sigma_{H} .
\end{aligned}
$$


To establish the next lemma we need the following auxiliary result.

Lemma 3.8. On a surface $\mathcal{S}$ as in Lemma 3.7, one has

$$
-Z \bar{\omega}=\mathcal{A}
$$

where $\mathcal{A}$ is the quantity defined in (3.6).

Proof. From the definition of $\bar{\omega}$ one has

$$
-Z \bar{\omega}=\bar{\omega} \frac{Z W}{W}-\frac{Z \omega}{W} .
$$

We now claim that

$$
\frac{Z \omega}{W}=\bar{q} T \bar{p}-\bar{p} T \bar{q}
$$

and that, furthermore,

$$
\frac{Z W}{W}=\bar{q} Y \bar{p}-\bar{p} Y \bar{q}+\bar{\omega} .
$$

It should be obvious to the reader that, inserting (3.11), (3.12) into (3.10), we obtain the desired conclusion. We are thus left with proving (3.11) and (3.12). For the former, we observe that

$$
<Z, N>=0 \text {. }
$$

If $\phi$ denotes a local defining function of $\mathcal{S}$ in the neighborhood of an arbitrary point, we thus have $Z \phi=0$. Applying $T$ to this identity, we obtain

$$
\begin{aligned}
0=T(Z \phi) & =T\left(\bar{q} X_{1} \phi-\bar{p} X_{2} \phi\right)=T \bar{q} X_{1} \phi+\bar{q} T X_{1} \phi-T \bar{p} X_{2} \phi-\bar{p} T X_{2} \phi \\
& =T \bar{q} X_{1} \phi-T \bar{p} X_{2} \phi+\bar{q} X_{1} T \phi-\bar{p} X_{2} T \phi=p T \bar{q}-q T \bar{p}+Z(T \phi)
\end{aligned}
$$

where we have used $\left[X_{i}, T\right]=0, i=1,2$. It follows that

$$
\frac{Z \omega}{W}=\frac{Z(T \phi)}{W}=\bar{q} T \bar{p}-\bar{p} T \bar{q},
$$

which proves (3.11). As for (3.12), we have

$$
\omega=T \phi=X_{1} X_{2} \phi-X_{2} X_{1} \phi=X_{1}(\bar{q} W)-X_{2}(\bar{p} W)=-\left(X_{2} \bar{p}-X_{1} \bar{q}\right) W+Z W,
$$

from which the desired conclusion follows immediately.

Using Lemma 3.5] in (3.5) of Corollary 3.4 in combination with Lemma 3.8, we obtain.

Lemma 3.9. Let $\mathcal{S} \subset \mathbb{H}^{1}$ be a $C^{3}$, oriented surface, with $\Sigma(\mathcal{S})=\varnothing$, then the second variation of the $H$-perimeter with respect to the deformation of $\mathcal{S}^{\lambda}=\mathcal{S}+\lambda h \boldsymbol{\nu}_{H}$, is given by

$$
\begin{aligned}
\mathcal{V}_{I I}^{H}\left(\mathcal{S} ; h \boldsymbol{\nu}_{H}\right) & =\int_{\mathcal{S}}\left\{(Z h)^{2}+h^{2}\left(2 \mathcal{A}-\bar{\omega}^{2}\right)\right\} d \sigma_{H} \\
& =\int_{\mathcal{S}}\left\{(Z h)^{2}+h^{2}\left[2(\bar{p} T \bar{q}-\bar{q} T \bar{p})+2 \bar{\omega}(\bar{q} Y \bar{p}-\bar{p} Y \bar{q})+\bar{\omega}^{2}\right]\right\} d \sigma_{H},
\end{aligned}
$$

where $\mathcal{A}$ is defined in (3.6). 
After these preparations we turn to the core of the proof of Theorem 1.2. We will focus on the case in which the surface is given by

$$
\mathcal{S}=\left\{(x, y, t) \in \mathbb{H}^{1} \mid x=y(\alpha t+\beta)\right\},
$$

the other family of surfaces $\mathcal{S}=\left\{(x, y, t) \in \mathbb{H}^{1} \mid y=x(\alpha t+\beta)\right\}$, with $\alpha<0$ and $\beta \in \mathbb{R}$, being treated by completely analogous considerations. Our first step in the proof of Theorem 1.2 is to compute the second variation of the $H$-perimeter for $\mathcal{S}$. In view of (3.9) in Lemma 3.7 or (3.13) in Lemma 3.9, we need to compute the quantities which appear as the coefficient of $a^{2}$ and $h^{2}$ in the integral in the right-hand side of the respective formulas. This is the content of the next lemma.

Lemma 3.10. Let $\mathcal{S}$ be the $H$-minimal surface given by (3.14), then one has

$$
\begin{gathered}
(\bar{p} T \bar{q}+\bar{q} T \bar{p})-\bar{\omega}(\bar{p} Y \bar{q}+\bar{q} Y \bar{p})-\bar{q}^{2} \bar{\omega}^{2} \\
-Z \bar{\omega}-\bar{p} \bar{q} \bar{\omega} \mathcal{H}=-\frac{2 \alpha}{W^{2}\left(1+(\alpha t+\beta)^{2}\right)}, \\
2(\bar{p} T \bar{q}-\bar{q} T \bar{p})+2 \bar{\omega}(\bar{q} Y \bar{p}-\bar{p} Y \bar{q})+\bar{\omega}^{2}=-\frac{2 \alpha}{W^{2}} .
\end{gathered}
$$

Proof. We can use the global defining function $\phi(x, y, t)=x-y(\alpha t+\beta)$. As previously stipulated, we assume that $\mathcal{S}$ is oriented in such a way that $\boldsymbol{N}=\nabla \phi=X_{1} \phi X_{1}+X_{2} \phi X_{2}+T \phi T$. Recalling (2.5), simple calculations based on (2.2) thus give

$$
p=X_{1} \phi=1+\frac{\alpha}{2} y^{2}, \quad q=X_{2} \phi=-\alpha t-\beta-\frac{\alpha}{2} x y, \quad \omega=T \phi=-\alpha y .
$$

The second equation in (3.17) becomes on $\mathcal{S}$

$$
q=-(\alpha t+\beta)\left(1+\frac{\alpha}{2} y^{2}\right) .
$$

We thus find on $\mathcal{S}$

$$
W^{2}=\left|\nabla^{H} \phi\right|^{2}=\left(1+\frac{\alpha}{2} y^{2}\right)^{2}\left(1+(\alpha t+\beta)^{2}\right) .
$$

Using (2.15) we obtain

$$
Z(T \phi)=\bar{q} X_{1}(T \phi)-\bar{p} X_{2}(T \phi)=\frac{\alpha p}{W}=\frac{\alpha\left(1+\frac{\alpha}{2} y^{2}\right)}{W}>0 .
$$

Next, we have

$$
X_{1} p=0, \quad X_{1} q=0, \quad X_{2} p=\alpha y, \quad X_{2} q=-\alpha x .
$$

This gives

$$
\left.X_{1} W=\frac{p X_{1} p+q X_{1} q}{W}=0, \quad X_{2} W=\frac{p X_{2} p+q X_{2} q}{W}=\alpha y\left(1+(\alpha t+\beta)^{2}\right)\right)^{1 / 2} .
$$

From (2.15) and (3.22) we find

$$
Z W=\bar{q} X_{1} W-\bar{p} X_{2} W=-\frac{p}{W} X_{2} W=-\alpha y .
$$

Combining (3.23) with (3.20) we obtain

$$
Z \bar{\omega}=\frac{Z(T \phi)}{W}-\frac{T \phi}{W^{2}} Z W=\frac{\alpha-\frac{\alpha^{2}}{2} y^{2}}{W^{2}} .
$$

Using the above formulas it is not difficult to verify that

$$
Y \bar{p}=Y \bar{q}=0 \text {. }
$$


We now have from 3.17)

$$
T W=\frac{p T p+q T q}{W}=-\frac{\alpha q}{W}
$$

and therefore we easily find

$$
\left\{\begin{array}{l}
T \bar{p}=-\frac{\alpha(\alpha t+\beta)}{\left(1+\frac{\alpha}{2} y^{2}\right)\left(1+(\alpha t+\beta)^{2}\right)^{3 / 2}}=-\frac{\alpha(\alpha t+\beta)}{W\left(1+(\alpha t+\beta)^{2}\right)}, \\
T \bar{q}=-\frac{\alpha}{W\left(1+(\alpha t+\beta)^{2}\right)}
\end{array}\right.
$$

From (3.17) and (3.26) we conclude that

$$
\bar{p} T \bar{q}+\bar{q} T \bar{p}=\frac{\alpha\left(1+\frac{\alpha}{2} y^{2}\right)\left((\alpha t+\beta)^{2}-1\right)}{W^{2}\left(1+(\alpha t+\beta)^{2}\right)} .
$$

From (3.18), (3.24), and (3.27), and elementary computations, we easily reach the conclusion that (3.15) holds. In a similar fashion, we obtain the proof of (3.16) by (3.17), (3.18), (3.19) and (3.26).

From Lemmas 3.7 3.9 and 3.10 we obtain the following corollary.

Corollary 3.11. Let $\mathcal{S}$ be the $H$-minimal surface given by (3.14). For any $a \in C_{0}^{\infty}(\mathcal{S})$, the second variation along the deformation $\mathcal{S} \rightarrow \mathcal{S}^{\lambda}=\mathcal{S}+\lambda a X_{1}$ is given by

$$
\mathcal{V}_{I I}^{H}\left(\mathcal{S} ; a X_{1}\right)=\int_{\mathcal{S}} \frac{\left(1+\frac{\alpha}{2} y^{2}\right)^{2}}{W^{2}}\left|\nabla^{H, \mathcal{S}} a\right|^{2} d \sigma_{H}-2 \alpha \int_{\mathcal{S}} \frac{a^{2}}{W^{2}\left(1+(\alpha t+\beta)^{2}\right)} d \sigma_{H} .
$$

For any $h \in C_{0}^{\infty}(\mathcal{S})$, the second variation along the deformation $\mathcal{S} \rightarrow \mathcal{S}^{\lambda}=\mathcal{S}+\lambda h \boldsymbol{\nu}_{H}$ is given by

$$
\mathcal{V}_{I I}^{H}\left(\mathcal{S} ; h \boldsymbol{\nu}_{H}\right)=\int_{\mathcal{S}}\left|\nabla^{H, \mathcal{S}} h\right|^{2} d \sigma_{H}-2 \alpha \int_{\mathcal{S}} \frac{h^{2}}{W^{2}} d \sigma_{H}
$$

We now consider the global smooth parametrization $\theta: \mathbb{R}^{2} \rightarrow \mathbb{R}^{3}$ of the surface $\mathcal{S}$ given by $\theta(y, t)=(y(\alpha t+\beta), y, t)$. Clearly, $\mathcal{S}=\theta\left(\mathbb{R}^{2}\right)$.

Lemma 3.12. Let $\mathcal{S}$ be the $H$-minimal surface given by (3.14). For any $a \in C_{0}^{\infty}(\mathcal{S})$, then one has

$$
\begin{aligned}
\mathcal{V}_{I I}^{H}\left(\mathcal{S} ; a X_{1}\right) & =\int_{\mathbb{R}^{2}} \frac{\left(1+\frac{\alpha}{2} y^{2}\right) u_{y}^{2}}{\left(1+(\alpha t+\beta)^{2}\right)^{3 / 2}} d y d t \\
& -2 \alpha \int_{\mathbb{R}^{2}} \frac{u^{2}}{\left(1+\frac{\alpha}{2} y^{2}\right)\left(1+(\alpha t+\beta)^{2}\right)^{3 / 2}} d y d t
\end{aligned}
$$

where $u=a \circ \theta \in C_{0}^{\infty}\left(\mathbb{R}^{2}\right)$. For any $h \in C_{0}^{\infty}(\mathcal{S})$, the one has

$$
\begin{aligned}
\mathcal{V}_{I I}^{H}\left(\mathcal{S} ; h \boldsymbol{\nu}_{H}\right) & =\int_{\mathbb{R}^{2}} \frac{\left(1+\frac{\alpha}{2} y^{2}\right) u_{y}^{2}}{\left(1+(\alpha t+\beta)^{2}\right)^{1 / 2}} d y d t \\
& -2 \alpha \int_{\mathbb{R}^{2}} \frac{u^{2}}{\left(1+\frac{\alpha}{2} y^{2}\right)\left(1+(\alpha t+\beta)^{2}\right)^{1 / 2}} d y d t
\end{aligned}
$$

where this time we have set $u=h \circ \theta \in C_{0}^{\infty}\left(\mathbb{R}^{2}\right)$. 
Proof. In order to prove (3.30) we make some reductions. Keeping in mind that from (2.23) we have $d \sigma_{H}=\left(\left|\nabla^{H} \phi\right| /|\nabla \phi|\right) d \sigma=(W /|\nabla \phi|) d \sigma$, from (3.19) we obtain

$$
\int_{\mathcal{S}} \frac{a^{2}}{W^{2}\left(1+(\alpha t+\beta)^{2}\right)} d \sigma_{H}=\int_{\mathbb{R}^{2}} \frac{u^{2}}{\left(1+\frac{\alpha}{2} y^{2}\right)\left(1+(\alpha t+\beta)^{2}\right)^{3 / 2}} d y d t .
$$

In order to express the first integral in the right-hand side of (3.28) as an integral on $\mathbb{R}^{2}$, we compute $\left|\nabla^{H, \mathcal{S}} a\right|^{2}$. We have from (2.9), (2.15), (3.17) and (3.18)

$$
\begin{aligned}
\left|\nabla^{H, \mathcal{S}} a\right|^{2} & =(Z a)^{2}=\left(\bar{q} X_{1} a-\bar{p} X_{2} a\right)^{2} \\
& =\frac{\left((\alpha t+\beta) X_{1} a+X_{2} a\right)^{2}}{1+(\alpha t+\beta)^{2}} .
\end{aligned}
$$

Now, the chain rule gives $u_{y}=(\alpha t+\beta) a_{x}+a_{y}$, and therefore we see from (2.2) that we have on $\mathcal{S}$

$$
(\alpha t+\beta) X_{1} a+X_{2} a=(\alpha t+\beta) a_{x}+a_{y}=u_{y} .
$$

From (3.32) we thus conclude that

$$
\int_{\mathcal{S}} \bar{p}^{2}\left|\delta_{H} a\right|^{2} d \sigma_{H}=\int_{\mathbb{R}^{2}} \frac{\left(1+\frac{\alpha}{2} y^{2}\right) u_{y}^{2}}{\left(1+(\alpha t+\beta)^{2}\right)^{3 / 2}} d y d t .
$$

This proves (3.30). The proof of (3.31) proceeds analogously, and we omit the details.

Lemma 3.13. Let $\chi_{k} \in C_{0}^{\infty}(\mathbb{R})$ be such that $0 \leq \chi_{k}(s) \leq 1, \chi_{k}(s)=0$ for $|s|>2 k, \chi_{k}(s) \equiv 1$ for $|s|<k$, and $\left|\chi_{k}^{\prime}(s)\right| \leq C / k$ with $C$ independent of $k$. Define for any $\alpha>0$

$$
f_{k}(y)=\frac{\chi_{k}(y)}{\sqrt{1+\frac{\alpha}{2} y^{2}}} .
$$

We have $f_{k} \in C_{0}^{\infty}(\mathbb{R})$, and there exists $k_{0} \in \mathbb{N}$ such that for all $k>k_{0}$

$$
\int_{\mathbb{R}} \frac{f_{k}(y)^{2}}{1+\frac{\alpha}{2} y^{2}} d y>\frac{1}{2 \alpha} \int_{\mathbb{R}}\left(1+\frac{\alpha}{2} y^{2}\right) f_{k}^{\prime}(y)^{2} d y .
$$

Proof. We begin by observing that integration of the right-hand side of (3.33) gives

$$
\begin{aligned}
\int_{\mathbb{R}} & \left(1+\frac{\alpha}{2} y^{2}\right) f_{k}^{\prime}(y)^{2} d y \\
= & \int_{\mathbb{R}}\left(\chi_{k}^{\prime}\right)^{2} d y+\frac{\alpha^{2}}{4} \int_{\mathbb{R}} \frac{y^{2} \chi_{k}^{2}}{\left(1+\frac{\alpha}{2} y^{2}\right)^{2}} d y+\frac{\alpha}{2} \int_{\mathbb{R}} \chi_{k}^{2}\left(\frac{y}{1+\frac{\alpha}{2} y^{2}}\right)^{\prime} d y
\end{aligned}
$$

Observing that

$$
\left(\frac{y}{1+\frac{\alpha}{2} y^{2}}\right)^{\prime}=\frac{1-\frac{\alpha}{2} y^{2}}{\left(1+\frac{\alpha}{2} y^{2}\right)^{2}},
$$

we conclude from (3.34) and dominated convergence

$$
\begin{aligned}
& \int_{\mathbb{R}}\left(1+\frac{\alpha}{2} y^{2}\right) f_{k}^{\prime}(y)^{2} d y=O\left(\frac{1}{k}\right)+\frac{\alpha}{2} \int_{\mathbb{R}} \frac{\chi_{k}^{2}}{\left(1+\frac{\alpha}{2} y^{2}\right)^{2}} d y \\
& \longrightarrow \frac{\alpha}{2} \int_{\mathbb{R}} \frac{1}{\left(1+\frac{\alpha}{2} y^{2}\right)^{2}} d y=\sqrt{\frac{\alpha}{2}} \frac{\pi}{2}, \quad \text { as } \quad k \rightarrow \infty .
\end{aligned}
$$


On the other hand, again by dominated convergence, we obtain for the integral in the left-hand side of (3.33)

$$
\begin{aligned}
& \int_{\mathbb{R}} \frac{f_{k}(y)^{2}}{1+\frac{\alpha}{2} y^{2}} d y=\int_{\mathbb{R}} \frac{\chi_{k}(y)^{2}}{\left(1+\frac{\alpha}{2} y^{2}\right)^{2}} d y \\
& \longrightarrow \int_{\mathbb{R}} \frac{1}{\left(1+\frac{\alpha}{2} y^{2}\right)^{2}} d y=\frac{\pi}{2} \sqrt{\frac{2}{\alpha}} \quad \text { as } \quad k \rightarrow \infty .
\end{aligned}
$$

In view of (3.35), (3.36), we obtain

$$
\int_{\mathbb{R}} \frac{f_{k}(y)^{2}}{1+\frac{\alpha}{2} y^{2}} d y-\frac{1}{2 \alpha} \int_{\mathbb{R}}\left(1+\frac{\alpha}{2} y^{2}\right) f_{k}^{\prime}(y)^{2} d y \longrightarrow \frac{3 \pi}{8} \sqrt{\frac{2}{\alpha}} .
$$

From this the conclusion readily follows.

As an immediate consequence of Lemma 3.13 and Fubini's theorem we obtain.

Corollary 3.14. For $k \in \mathbb{N}, k \geq k_{0}$, define $u_{k}(y, t)=f_{k}(y) \chi_{k}(t)$, where $\chi_{k}$ and $f_{k}$ and $k_{0}$ are as in Lemma 3.13, One has

$$
\int_{\mathbb{R}^{2}} \frac{u_{k}(y, t)^{2}}{\left(1+\frac{\alpha}{2} y^{2}\right)\left(1+(\alpha t+\beta)^{2}\right)^{3 / 2}} d y d y>\frac{1}{2 \alpha} \int_{\mathbb{R}^{2}} \frac{1+\frac{\alpha}{2} y^{2}}{\left(1+(\alpha t+\beta)^{2}\right)^{3 / 2}}\left(\frac{\partial u_{k}(y, t)}{\partial y}\right)^{2} d y d t
$$

We are finally ready to give the

Proof of Theorem 1.2. Let $u_{k}, k \geq k_{0}$, be as in Corollary 3.14. Define $a_{k}: \mathbb{H}^{1} \rightarrow \mathbb{R}, a_{k} \in$ $C_{0}^{\infty}\left(\mathbb{H}^{1}\right)$, as follows

$$
a_{k}(x, y, t)=\frac{\chi_{k}(y) \chi_{k}(t) \chi_{k}(x-y(\alpha t+\beta))}{\sqrt{1+\frac{\alpha}{2} y^{2}}} .
$$

We observe that $a_{k}(\theta(y, t))=u_{k}(y, t) \chi_{k}(0)=u_{k}(y, t)$. At this point, appealing to (3.30) in Lemma 3.12 and to Corollary 3.14 we conclude that for every fixed $k \geq k_{0}$, we have for the deformation $\mathcal{S} \rightarrow \mathcal{S}^{\lambda}=\mathcal{S}+\lambda a_{k} X_{1}$

$$
\mathcal{V}_{I I}^{H}\left(\mathcal{S} ; a_{k} X_{1}\right)=\left.\frac{d^{2}}{d \lambda^{2}} P_{H}\left(\mathcal{S}^{\lambda}\right)\right|_{\lambda=0}<0
$$

This proves that $\mathcal{S}$ cannot be a local minimizer of the $H$-perimeter for compactly supported deformations along $X_{1}$. In a similar way, using (3.30) in Lemma 3.12 and Corollary [3.14 we see that $\mathcal{S}$ cannot be a local minimizer for deformations along the horizontal normal $\boldsymbol{\nu}_{H}$. In particular, since every global minimizer is also a local one, $\mathcal{S}$ cannot be a global minimizer either.

\section{REFERENCES}

[A] L. Ambrosio, Some fine properties of sets of finite perimeter in Ahlfors regular metric measure spaces, Adv. Math., 159 (2001), 51-67.

[AK1] L. Ambrosio \& B. Kircheim, Rectifiable sets in metric and Banach spaces, Math. Ann., 318 (2000), $527-555$. [AK2] _ Currents in metric spaces, Acta Math., 185 (2000), 1-80.

[ASV] L. Ambrosio, F. Serra Cassano \& D. Vittone, Intrinsic regular hypersurfaces in Heisenberg groups, preprint, 2005.

[B] Z. M. Balogh, Size of characteristic sets and functions with prescribed gradients, J. Reine Angew. Math., $\mathbf{5 6 4} 2003,63-83$.

[BRS] Z. M. Balogh, M. Rickly \& F. Serra Cassano, Comparison of Hausdorff measures with respect to the Euclidean and the Heisenberg metric, Publ. Math., 47 (2003), 237-259.

[Be] A. Bellaïche, The tangent space in sub-Riemannian geometry. Sub-Riemannian geometry,, Progr. Math., 144 (1996), Birkhäuser, 1-78. 
[BC] M. Bonk \& L. Capogna, Horizontal mean curvature flow in the Heisenberg group, preprint, 2005.

[CDG] L. Capogna, D. Danielli \& N. Garofalo, The geometric Sobolev embedding for vector fields and the isoperimetric inequality, Comm. Anal. and Geom., 2 (1994), 201-215.

[CG] L. Capogna \& N. Garofalo, Ahlfors type estimates for perimeter measures in Carnot-Carathéodory spaces, preprint, 2004.

[Ca] C. Carathéodory, Calculus of Variations, third edition, Chelsea Publ. Co., New York, 1989.

[CH] J.H.Cheng \& J.F. Hwang, Properly embedded and immersed minimal surfaces in the Heisenberg group, Bull. Austral. Math. Soc., 70 (2004), no. 3, 507-520.

[CHMY] J.H. Cheng, J. F. Hwang, A. Malchiodi \& P. Yang, Minimal surfaces in pseudohermitian geometry and the Bernstein problem in the Heisenberg group, revised version 2004, Ann. Sc. Norm. Sup. Pisa, 1 (2005), 129-177.

[CS1] G. Citti and A. Sarti, Subjective surfaces and Riemannian mean curvature flow of graphs, Acta Math. Univ. Comenian. (N.S.) $\mathbf{7 0}$ (2000), no. 1, 85-103.

[CS2] — A cortical based model of perceptual completion in roto-translation space, preprint, 2004.

[CMS] G. Citti, and M. Manfredini \& A. Sarti, From neural oscillations to variational problems in the visual cortex, Journal of Physiology-Paris, 97 (2003), 379-385.

[CM] T. H. Colding \& W. P.Minicozzi II, Minimal Surfaces, Courant Lec. Notes in Mathematics, 4, 1999.

[DGN1] D. Danielli, N. Garofalo \& D. M. Nhieu, Trace inequalities for Carnot-Carathéodory spaces and applications, Ann. Sc. Norm. Sup. Pisa, Cl. Sci. (4), 2, 27 (1998), 195-252.

[DGN2] - Hypersurfaces of minimal type in sub-Riemannian geometry, Proc. of the meeting "Second Order Subelliptic Equations and Applications", Cortona 2003, Lecture Notes S.I.M., E. Barletta, ed., 2005.

[DGN3] — Sub-Riemannian calculus on hypersurfaces in Carnot groups, preprint, 2006.

[DGN4] _ Non-doubling Ahlfors measures, Perimeter measures, and the characterization of the trace spaces of Sobolev functions in Carnot-Carathéodory spaces, Memoirs of the Amer. Math. Soc., vol.182, 2006, p.119.

[DGN5] _ A partial solution of the isoperimetric problem for the Heisenberg group, Forum Math., to appear.

[DS] G. David \& S. Semmes, Fractured fractals and Broken Dreams: Self-Similar Geometry through Metric and Measure, Oxford Lecture Series in Mathematics and Its Applications, Clarendon Press, Oxford, 1997.

[DG1] E. De Giorgi, Su una teoria generale della misura $(r-1)$-dimensionale in uno spazio a $r$ dimensioni, Ann. Mat. Pura Appl., 36 (1954), 191-213.

[DG2] - Nuovi teoremi relativi alla misura $(r-1)$-dimensionale in uno spazio a $r$ dimensioni, Ric. Mat., 4 (1955), 95-113.

[DCP] E. De Giorgi, F. Colombini \& L. C. Piccinini, Frontiere orientate di misura minima e questioni collegate, Sc. Norm. Sup. Pisa, Cl. Scienze, Quaderni, 1972.

[E1] P. Eberlein, Geometry of 2-step nilpotent groups with a left invariant metric, Ann. Sci. cole Norm. Sup. (4) 27 (1994), no. 5, 611-660.

[E2] - Geometry of 2-step nilpotent groups with a left invariant metric. II, Trans. Amer. Math. Soc., 343 (1994), no. 2, 805-828.

[E3] - Geometry of nonpositively curved manifolds, Chicago Lectures in Mathematics. University of Chicago Press, Chicago, IL, 1996.

[Fo] G. B. Folland, Subelliptic estimates and function spaces on nilpotent Lie groups, Ark. Math., 13 (1975), 161-207.

[FSS1] B. Franchi, R. Serapioni \& F. Serra Cassano, Rectifiability and perimeter in the Heisenberg group, Math. Ann., 321 (2001) 3, 479-531.

[FSS2] _ Regular hypersurfaces, intrinsic perimeter and implicit function theorem in Carnot groups, Comm. Anal. Geom., 11 (2003), no. 5, 909-944.

[FSS3] _ On the structure of finite perimeter sets in step 2 Carnot groups, J. Geom. Anal., 13 (2003), no. 3, 421-466.

[FSS4] _ Regular submanifolds, graphs and area formulas in Heisenberg groups, preprint, 2004.

[GN] N. Garofalo \& D. M. Nhieu, Isoperimetric and Sobolev inequalities for Carnot-Carathéodory spaces and the existence of minimal surfaces, Comm. Pure Appl. Math., 49 (1996), 1081-1144.

[GP] N. Garofalo \& S. D. Pauls, The Bernstein problem in the Heisenberg group, preprint, 2003.

[GS] N. Garofalo \& C. Selby, Equations of mean curvature type in the Heisenberg group, work in progress, 2005.

[G] E. Giusti, Minimal surfaces and functions of bounded variation, Birkhäuser, 1984.

[Gro] M. Gromov, Carnot-Carathéodory spaces seen from within, in Sub-Riemannian Geometry, Progress in Mathematics, vol. 144, edited by André Bellä̈che \& Jean-Jacques Risler, Birkhäuser, 1996.

[HP1] R. K. Hladky \& S. D. Pauls, Minimal surfaces in the roto-translation group with applications to a neurobiological image completion model, preprint, 2005.

[HP2] R. K. Hladky \& S. D. Pauls, Constant mean curvature surfaces in sub-Riemannian geometry, preprint, 2005. 
[KR] A. Korányi \& H. M. Reimann, Quasiconformal mappings on the Heisenberg group, Invent. Math., 80 (1985), no. 2, 309-338.

[LM] G. P. Leonardi \& S. Masnou, On the isoperimetric problem in the Heisenberg group $\mathbb{H}^{n}$, Ann. Mat. Pura Appl., (4) 184 (2005), no. 4, 533-553.

[LR] G. P. Leonardi \& S. Rigot, Isoperimetric sets on Carnot groups, Houston J. Math., 29 (2003), no. 3, $609-637$.

[Ma1] V. Magnani, Differentiability and area formula on stratified Lie groups, Houston J. Math., 27 (2001), 297-323.

[Ma2] _ A blow-up theorem for regular hypersurfaces on nilpotent groups, Manuscripta Math., 110 (2003), $55-76$.

[Ma3] - Characteristic points, rectifiability and perimeter measure on stratified groups, preprint, 2004.

[MM] U. Massari \& M. Miranda, Minimal Surfaces of Codimension One, Math. Studies 91, North-Holland, 1984.

[Mo] F. Montefalcone, Hypersurfaces and variational formulas in sub-Riemannian Carnot groups, preprint, 2006.

[MSC] R. Monti \& F. Serra Cassano, Surface measures in Carnot-Carathéodory spaces, Calc. Var., 13 (2001), 339-376.

[Pa1] P. Pansu, Une inǵalité isopérimétrique sur le groupe de Heisenberg, C. R. Acad. Sci. Paris Sér. I Math., 295 (1982), no. 2, 127-130.

[Pa2] - Métriques de Carnot-Carathéodory et quasi-isométries des espaces symétriques de rang un, Ann. of Math. (2)129 (1989), 1-60.

[P1] S. Pauls, Minimal surfaces in the Heisenberg group, Geom. Dedicata, 104 (2004), 201-231.

[P2] _ A notion of rectifiability modelled on Carnot groups, Indiana U. Math. J., 53 (2004), 49-81.

[RR] M. Ritorè \& C. Rosales, Rotationally invariant hypersurfaces with constant mean curvature in the Heisenberg group $\mathbb{H}^{n}$, preprint, April 2005.

[Se1] C. Selby, An extension and trace theorem for functions of $H$-bounded variation in Carnot groups of step 2, Houston J. Math., to appear.

[Se2] _ Geometry of hypersurfaces in Carnot groups of step 2, Ph. D. Dissertation, Purdue University, 2006.

[S] E. M. Stein, Harmonic Analysis: Real Variable Methods, Orthogonality and Oscillatory Integrals, Princeton Univ. Press, (1993).

Department of Mathematics, Purdue University, West Lafayette, IN 47907

E-mail address, Donatella Danielli: danielli@math.purdue.edu

Department of Mathematics, Purdue University, West Lafayette, IN 47907

E-mail address, Nicola Garofalo: garofalo@math.purdue.edu

Department of Mathematics, Georgetown University, Washington, DC 20057-1233

E-mail address, Duy-Minh Nhieu: nhieu@math.georgetown.edu 